$\begin{array}{cl}\begin{array}{c}\text { Revue } \\ \text { de /histoire } \\ \text { des religions }\end{array} & \text { Revue de l'histoire des religions } \\ & 1 \mid 2010 \\ \text { Beauté du rite }\end{array}$

\title{
Retrouver la foi par la beauté : réalité et utopie du mouvement néogothique dans l'Europe du XIX siècle
}

Finding Faith Through Beauty: Reality and Utopia of the Neo-Gothic Movement in $19^{\text {th }}-C$ Europe

\section{Bernard Berthod}

\section{OpenEdition \\ Journals}

Édition électronique

URL : http://journals.openedition.org/rhr/7563

DOI : $10.4000 /$ rhr.7563

ISSN : $2105-2573$

Éditeur

Armand Colin

Édition imprimée

Date de publication : 1 mars 2010

Pagination : 75-92

ISBN : 978-2200-92612-0

ISSN : 0035-1423

\section{Référence électronique}

Bernard Berthod, «Retrouver la foi par la beauté : réalité et utopie du mouvement néogothique dans I'Europe du XIX`e siècle », Revue de l'histoire des religions [En ligne], 1 | 2010, mis en ligne le 01 mars 2013, consulté le 20 avril 2019. URL : http://journals.openedition.org/rhr/7563 ; DOI : 10.4000/ rhr.7563 


\section{Retrouver la foi par la beauté : réalité et utopie du mouvement néogothique dans l'Europe du XIX ${ }^{\mathrm{e}}$ siècle}

Le mouvement néogothique en Europe occidentale (France, Angleterre, Allemagne) trouve un écho spirituel auprès de quelques hommes d'Églises et prélats qui fondent dans cette vision romantique du Moyen Âge l'espoir de rechristianiser leurs pays. Quelques prélats fortunés font bâtir des églises et réaliser des vases sacrés et des vêtements liturgiques dans le goût médiéval, aidés dans leur réflexion et leur choix par des théoriciens, des architectes et des éditeurs. Leurs idées et leur enthousiasme sont mal compris à Rome. En fait, le mouvement archéologique n'aura pas un grand impact sur l'ensemble des fidèles, touchant seulement l'élite intellectuelle. Cependant, la forme des vêtements et des vases sacrés restera acquise au $X X^{e}$ siècle.

\section{Finding Faith Through Beauty : Reality and Utopia of the Neo-Gothic Movement in $19^{\text {th }}-\mathrm{C}$ Europe}

The Neo-Gothic movement in Western Europe (France, England, Germany) found spiritual resonance among some churchmen and prelates who based their hope of rechristianizing their countries on this romantic vision of the Middle Ages. Some wealthy prelates had churches built and ordered sacred vases and liturgical vestments in the medieval taste, helped in their reflection and choices by theorists, architects and editors. Their ideas and enthusiasm were misunderstood in Rome. In fact, the archaeological movement did not have a profound impact on the faithful but only reached the intellectual elite. However, the Neo-Gothic influence on the forms of vestments and of sacred vessels was maintained throughout the $20^{\text {th }}$ century. 
Le siècle des Lumières ne s'est guère intéressé à l'art liturgique. La paramentique est souvent taillée dans des étoffes civiles sans même de modestes broderies pour lui donner une connotation chrétienne. Dès 1805 , la situation évolue rapidement grâce à la réorganisation de l'Église de France accompagnée d'un grand mouvement pro-chrétien suscité par Chateaubriand. La pratique religieuse reprend vigueur et les pèlerinages sont rendus plus aisés grâce au développement du chemin de fer. Le clergé se romanise et les liturgies néo-gallicanes sont abandonnées entre 1840 et 1853 pour une liturgie romaine unifiée (encyclique Inter Multiplices de 1853). Cette renaissance est marquée par deux événements romains : la proclamation triomphale du dogme de l'Immaculée Conception par Pie IX, le 8 décembre 1854, et la réunion du Concile du Vatican en décembre 1869, auquel assistent tous les évêques européens même si plus d'un tiers d'entre eux ne sont pas ultramontains. Ces deux moments permettent aux membres de la hiérarchie de se connaître en dépassant les limites de leur diocèse. Le Concile est l'occasion d'une exposition d'art liturgique à laquelle contribuent tous les grands fournisseurs européens.

Le mouvement néogothique auquel adhère l'élite ultramontaine prône l'apostolat par la Beauté. Selon ses partisans, la liturgie et son décor doivent être purifiés pour retrouver la simplicité et la grandeur du siècle de saint Louis. Les changements sont plus visibles pour la paramentique que pour l'orfèvrerie : le vêtement liturgique subit une véritable mutation avec l'emploi du tissu à décor médiéval et le retour de l'orfroi brodé et des formes amples, il perd sa rigidité due à la doublure en bougran ${ }^{1}$; la chasuble redevient l'antique planète en forme de cercle ou d'ovale, la dalmatique retrouve des manches et la mitre s'abaisse. La broderie, reprenant les grands décors hagiographiques, permet la mise en scènes des saints locaux qui ont disparu des propres diocésains ${ }^{2}$ avec l'imposition de la liturgie romaine. Portée par cet élan, la production liturgique se développe d'une manière exponentielle jusqu'à la fin du siècle.

1. Toile de chanvre gommée et rigide qui sert de soutien aux étoffes souples.

2. Textes liturgiques spécifiques à chaque diocèse. 


\section{LES ARTISANS DU RENOUVEAU}

Parmi les précurseurs qui travaillèrent au renouveau de l'art liturgique selon les normes néogothiques, cinq nous paraissent essentiels : ce sont trois ecclésiastiques, Dom Guéranger, Arthur Martin et Franz Bock; un architecte, August Pugin; et un archéologue, Adolphe Napoléon Didron ${ }^{3}$.

Prosper Guéranger, né le 4 avril 1805, à Sablé, dans la Sarthe, ordonné prêtre à 22 ans, devient le secrétaire de l'évêque du Mans, Mgr de la Myre-Mory; il le suit à Paris, en 1829, lors de la retraite de ce dernier et s'y fixe. Il rencontre plusieurs catholiques dont les tendances antigallicanes lui plaisent. Dès cette époque, il rêve de restaurer, en France, l'ordre de Saint-Benoît. En 1833, avec quelques compagnons, il s'installe dans les bâtiments d'un ancien monastère mauriste de Sablé. Il écrit plusieurs ouvrages où il expose sa conception de la liturgie et ses vues ultramontaines. Dans le Mémorial catholique, revue mennaisienne à laquelle il collabore, il écrit, en juillet 1830, que le mouvement liturgique appelle une régénération totale des formes reposant sur des sciences exactes: l'archéologie et l'histoire documentaire ${ }^{4}$. "Après s'être préalablement débarrassés de l'amas de nouveautés dont le $\mathrm{XVIII}^{\mathrm{e}}$ siècle a encombré la liturgie, nos diocèses devraient rentrer dans la forme romaine et reprendre les prières qu'ils ont reçues au temps de Charlemagne 5 . » Cette vision "poétique» de la liturgie déclenche une tempête au sein du clergé et de l'épiscopat, que Dom Guéranger qualifie de "véritable révolution liturgique ${ }^{6}$ ». Il publie en 1840 les Institutions liturgiques dans lesquelles il souligne la difficulté de faire comprendre aux fidèles « l'intelligence des mystères, si ample à la fois et si facile par la liturgie, ravalée pour la plupart des fidèles à la mesure de ces innombrables petits livres qui inondent de plus en plus la librairie religieuse ». Il tente de démontrer que la liturgie romaine représente la forme originale du culte ayant survécu depuis les temps apostoliques. En 1841, il

3. Bernard Berthod, Élisabeth Hardouin Fugier, Paramentica, tissus lyonnais et art sacré, 1800-1914, Lyon, 1990, p. 41-56; et Dictionnaire des arts liturgiques, Paris, 1996, aux noms cités.

4. Hameline, « Viollet-le-Duc », La Maison-Dieu, Paris, 1969, p. 76.

5. Prosper Guéranger, Institutions liturgiques, Paris, 1841, t. II, p. 636.

6. Ibid., p. 693. 
entreprend la rédaction de son ouvrage fondamental, L 'Année liturgique, dont le but est de faire connaître aux catholiques le sens et le goût des textes liturgiques et des rites en donnant des explications historiques et en soulignant leur beauté ${ }^{7}$. Il écrit dans la préface générale : « Le Moyen Âge des Églises d'Occident a produit, dans le genre liturgique, des séquences d'une rare beauté; un de nos premiers soins sera d'initier les fidèles qui nous liront à ces sources si pures de tendresse et de rêve ${ }^{8}$. \ Le savant bénédictin dessine des croquis de chasubles en situation, conservés aujourd'hui à Solesmes, qui montrent l'abbé dans diverses fonctions pontificales ainsi que des chasubles amples. Il commande, à Reims, la copie d'une chasuble conservée au Trésor de la cathédrale ${ }^{9}$. Il rencontre beaucoup de résistance, le cardinal d'Astros l'attaque violemment dans un pamphlet, L'Église de France injustement flétrie (1843). Petit à petit, quelques évêques prennent conscience de l'intérêt de ses travaux et le soutiennent. En 1850, recommandé par l'évêque de Poitiers, Mgr Pie, il devient consulteur de la Sacrée Congrégation des Rites. La restauration liturgique reste liée au nom de Dom Guéranger, même si le mouvement entraîne l'abandon d'antiques coutumes auxquelles la France était «obstinément demeurée fidèle ».

Auguste-Charles Pugin, né en 1812, à Londres, commence très tôt sa carrière d'architecte en adhérant avec enthousiasme au Gothic Revival. Anglican, il se convertit au catholicisme en 1835 et commence à dessiner des objets de culte et des vêtements liturgiques. Dès 1838, il écrit dans l'Orthodox Journal : « The dignity of vestments depends principally on their form. Without flowing lines and grand easy folds no majestic appearance can be obtained. The old chasubles, unpinched in shape fell gracefully from the schoulders and folded avec the armes, pliant material ${ }^{10}$. " En 1844, il publie

7. 9 volumes paraissent chez Oudin à Paris de 1841 à 1866.

8. Prosper Guéranger, L'année liturgique, t. I, Paris, 1844, p. 23.

9. Cette chasuble, publiée par F. Bock dans Geschichte der liturgischen Gewänder des Mittelalters oder Entstechung und Entwicklung der Kirchlichen Ornate und Paramente, Bonn, 1859, t. II, pl. 12. Les religieuses de 1'Enfant-Jésus de Cologne en réalisent quelques exemplaires. Celle de Dom Guéranger est conservée à Solesmes, $c f$. B. Berthod, Fils de soie, Chambord, 1994, p. 50.

10. Auguste-Charles Pugin, " Chasuble of Cloth of gold, embroidered », The London and Dublin Orthodox Journal of Useful Knowledge, $\mathrm{n}^{\circ} 146$, avril 1838, p. 227. Cité par Bede Millard, Pugin, a Gothic Passion, Londres, 1994, p. 208. 
un dictionnaire du costume ecclésiastique et de son ornementation : The Glossary of Ecclesiastical Ornments and Costume. Il fait exécuter les objets d'orfèvrerie par la firme londonienne John Hardmann \& Co, à laquelle il s'associe en 1838. Après sa mort en 1852, la firme Hardmann \& Co continuera à commercialiser ses créations, fournissant, parmi d'autres, les chapelles prélatices des cardinaux Newman et Manning, archevêque de Westminster ${ }^{11}$.

Le jésuite Arthur Martin rassemble un grand nombre de décors, relevés par lui-même sur des monuments d'architecture, des objets sacrés et des étoffes. Il publie ce corpus dans les Mélanges archéologiques, dès 1847. Après sa disparition, en 1856, le père Cahier, jésuite et fils du célèbre orfèvre parisien, prend la relève en publiant la Suite aux Mélanges d'Archéologie, en 1868. À partir de sa documentation, il dessine un grand nombre de vêtements liturgiques : « Pour gagner l'argent qu'il dépensait à publier les plus savants mémoires qui aient été imprimés sur le Moyen-Âge, il donnait aux orfèvres et aux brodeurs des modèles ${ }^{12}$. » Le chanoine Bock, qui sera son émule, écrit qu'il sut allier la théorie et la pratique : «Il composait de savants traités sur la forme primitive des vêtements sacrés, en même temps il dessinait des modèles pleins de grâce et de noblesse, destinés à décorer les chasubles, à les remettre en rapport avec l'esprit et les sentiments qui animèrent l'art chrétien dans les beaux jours du XIII ${ }^{e}$ siècle $^{13}$. $\gg$ De plus, membre de la commission des Arts et Édifices religieux, il intervient dans les pourparlers entre le gouvernement et les diocèses demandant le financement de vêtements liturgiques et d'orfèvrerie sacrée. Il n'hésite pas à écrire à de modestes chasublières provinciales, pour les persuader d'adhérer au mouvement archéologique. Ainsi cette lettre à Thérèse Guichon, installée à Albi, qui doit honorer une commande du Ministère pour la cathédrale Sainte-Cécile et qui lui soumet son projet dans le style français (1851) :

On abandonne presque partout, aujourd'hui, cette forme étriquée $\mathrm{du} \mathrm{XVIII}^{\mathrm{e}}$ siècle pour se rapprocher de l'usage romain ou de l'usage des anciens fidèles. J'ai indiqué au crayon sur le dessin la légère réforme

11. Susan Foister, Cardinal Newman, 1801-1890, Londres, 1990, p. 58.

12. Alfred Dancel, «Renaissance de l'orfèvrerie du Moyen Âge », Gazette des beaux-arts, 1888, p. 225.

13. Franz Bock, «Les vêtements sacerdotaux dans l'Allemagne moderne», Revue de l'art chrétien, 1861, p. 94-95. 
que je crois devoir conseiller [...]. Je trouve qu'il serait mieux de fermer par des cordons les manches des dalmatiques qui finiront par perdre entièrement leur caractère ${ }^{14}$.

Franz Bock, chanoine de la cathédrale de Cologne, a été très marqué par les travaux d'Arthur Martin et de Charles de Linas. Grand collectionneur de tissus médiévaux, il recueille dans toute l'Europe plus de 1500 échantillons de tissu et broderie du Moyen Âge. Il édite en 1859 à Bonn, un glossaire détaillé du vestiaire liturgique, en trois tomes, avec patron de chaque élément ${ }^{15}$; puis en 1860 à Cologne, chez Veber \& Deckers, un recueil de lithographies de dessins qui eurent beaucoup de succès auprès des fabricants. Ces ouvrages ont eu un grand retentissement en Allemagne, auprès de plusieurs évêques et de nombreux prêtres; le cardinal de Cologne, les évêques de Munster et d'Osnabrück adoptèrent les vêtements pontificaux de forme ample dès 1860. La collection de Franz Bock, dispersée dans de nombreux musées européens (Victoria \& Albert, de Londres, Musée des Tissus de Lyon, Kunsthistorisches Museum de Vienne, Nuremberg et Paris) permit une meilleure connaissance de visu du tissu médiéval et fut un des facteurs du renouveau du vêtement liturgique, ainsi que le note Louis de Farcy ${ }^{16}$.

Adolphe-Napoléon Didron, jeune archéologue passionné par le Moyen Âge, n'a pas eu en France la place qu'il méritait; peu diplomate, il ne sut pas trouver les appuis politiques dont il aurait eu besoin pour mener à bien son grand projet de restauration du parc immobilier médiéval français. Il a cependant, à force de travail et d'opiniâtreté, entretenu un important réseau de correspondance avec tout ce que l'Europe comptait d'architectes et d'érudits adeptes du mouvement archéologique. À partir de 1844, il édite les Annales archéologiques; les vingt-sept tomes ont été pour tous les amateurs, artistes, artisans de province une source d'inspiration inépuisable ${ }^{17}$. Au fil des volumes, les grands thèmes de l'iconogra-

14. Christine Aribaud, «L'étoffe des solennités : la soie en service dans les églises du Tarn ( $\mathrm{Xv}^{\mathrm{e}}-\mathrm{Xx} \mathrm{e}^{\mathrm{e}}$ siècle) », Textiles sacrés du Tarn, Paris, 2003, p. 35.

15. Geschichte der liturgischen Gewänder des Mittelalters oder Entstechung und Entwicklung der Kirchlichen Ornate und Paramente, Bonn, 1859.

16. Louis de Farcy, La Broderie du onzième siècle jusqu'à nos jours, Angers, 1890, p. 111.

17. Catherine Brissac, Jean-Michel Leniaud, « Adolphe Napoléon Didron ou les médias au service de l'art chrétien », Revue de l'art, Paris, 1987, n 77 , p. 3342. 
phie médiévale sont développés en insistant sur la dimension symbolique; par exemple le tétramorphe ${ }^{18}$, la symbolique des pierres, celle des fleurs à propos de l'ostensoir dessiné par l'abbé Pouguet pour Saint-Vincent de Marseille ${ }^{19}$. Le vêtement liturgique est évoqué par Didron lui-même, s'appuyant sur un texte de saint Jérôme pour justifier la « richesse » des vêtements. L'auteur décrit minutieusement l'emploi des couleurs selon Innocent III et leur symbolisme $^{20}$.

\section{LES ULTRAMONTAINS}

L'image du pape ne cesse de grandir en France sous la plume d'un Chateaubriand ou d'un Joseph de Maistre. Le contraste est grand entre la catholicité romaine, puissante et unifiée depuis le retour triomphal de Pie VII dans ses États (1815), et l'expression liturgique française perçue comme sans grandeur et disparate. En 1800 (avant le Concordat), sur cent trente diocèses d'Ancien Régime, plus de quatre-vingt pratiquent une liturgie néo-gallicane proche du jansénisme et douze seulement suivent le rite romain. La réorganisation concordataire des diocèses (avec suppression de près de cinquante anciens) porte à son comble la confusion liturgique. C'est ainsi qu' au diocèse de Langres, se rencontrent et interfèrent les liturgies de Toul, de Châlons-sur-Marne, de Troyes, de Besançon et de Langres, la ville épiscopale ${ }^{21}$. Les pasteurs deviennent conscients de l'urgence d'une réforme et d'un retour à l'unité. Ce retour est rendu difficile par la faiblesse des études ecclésiastiques. Un essai non concluant des gallicans se met en place autour de la liturgie parisienne. L'unité autour de la liturgie romaine est imposée par l'encyclique Inter multiplices de 1853. Dès les années 1840, dans chaque diocèse, quelques prêtres ultramontains, souvent jeunes et

18. Félicie d'Arzac, Annales archéologiques, t. VIII, p. 205-215.

19. Adolphe Nicolas Didron, Annales archéologiques, t. XXVII, 1870, p. $56-103$.

20. Op. cit., t. IV, p. 363-368.

21. Voir Gérard Cholvy, Histoire religieuse de la France contemporaine, Paris, t. I, 1985 : «Ce n'est pas de diocèse à diocèse que s'affrontent les missels mais de paroisse à paroisse; il n'est pas rare de trouver dans le même diocèse, des prêtres suivant trois ou quatre liturgies différentes, celles de leur ancienne circonscription ecclésiastique. » 
enthousiastes, stimulent le reste du corps clérical et aiguillonnent leur évêque. Férus d'archéologie, ils idéalisent à l'extrême la liturgie romaine et adhèrent au mouvement néogothique. Pour eux la foi du siècle de saint Louis est une réalité qu'ils veulent faire revivre. «Nous ne rêvions, écrit dans ses mémoires l'abbé Haigneré, qu'architecture gothique, vêtements sacerdotaux du moyen âge, chant romain $\mathrm{du} \mathrm{XIII}^{\mathrm{e}}$ siècle et restauration des ordres religieux ${ }^{22}$. » Ces prêtres sont les clients tous désignés des chasubliers et des orfèvres qui s'adaptent à leur demande.

Le courant ultramontain est encouragé par les congrégations qui se réinstallent en Europe et les instituts nouvellement créés, telles les missionnaires du Précieux Sang, fondés par Gaspard de Buffalo en 1815, les oblats de Marie Immaculée, par Eugène de Mazenod en 1816, les maristes par l'abbé Collin en 1816, les marianistes par le chanoine Jacques Chaminade en 1817, les pallotins par Vicenzo Pallotino à Rome en 1835, les augustins de l'Assomption par Emmanuel d'Alzon, en 1845, les chanoines réguliers de l'Immaculée-Conception par Dom Gréa en 1866, les missionnaires de Saint-Joseph de Mill Hill à Londres en 1866 et bien d'autres. En 1853, à Rome, un séminaire français, confié aux spiritains ${ }^{23}$, ouvre ses portes via Santa Chiara, permettant à l'élite du jeune clergé français de devenir vraiment romaine. Dans le même temps les collèges belge et teutonique ouvrent leurs portes à l'élite ecclésiastique de leurs principautés.

Les cérémonies romaines de la proclamation du dogme de l'Immaculée Conception, le 8 décembre 1854, par le rassemblement épiscopal sans précédent qu'elles suscitent, marquent un tournant décisif pour l'ultramontanisme encore accru par la convocation du concile du Vatican, en décembre 1869. Les dernières années du pontificat et la célébration du jubilé de Pie IX (1877) entraînent l'adhésion de l'ensemble de l'épiscopat, y compris des derniers gallicans. Dès lors les pèlerinages vers la Ville éternelle se multiplient, profitant du développement des chemins de fer (achèvement du tunnel ferroviaire sous le mont Cenis en 1871). Les premiers pèlerinages ouvriers sous la conduite d'Albert de

22. Cité par Austin Gough, Paris et Rome, les catholiques français et le pape au XIXe siècle, Paris, 1996, p. 157.

23. Congrégation de prêtres fondée par François Libermann en 1841. 
Mun et de Léon Hammel arrivent sous le pontificat de Léon XIII. L'influence artistique italienne traditionnelle se trouve renforcée par les échanges spirituels et les arts liturgiques sont dynamisés par le contexte ultramontain.

\section{LA MIE EN GLURE PAR QUELLUES PRELLAT FRANGAIS}

Le terrain est favorable à un renouveau liturgique car il règne une grande misère spirituelle dans les paroisses. De nombreuses relations de voyages s'en font l'écho, comme cette procession de la Fête-Dieu à Montpellier « avec un clergé pauvrement vêtu, peu nombreux et sans ornement ${ }^{24} \gg$. Mais le décor et la somptuosité des vêtements et des vases que l'on rencontre en France à la fin du règne de Louis-Philippe ne suffisent pas. Il faut un souffle nouveau, une animation de l'intérieur. Un vicaire de Paris écrit à son archevêque, en 1849 :

Si le culte public consiste en riches et nombreux ornements [...] alors, il faut l'avouer, le culte est noble et imposant à Paris. Mais si le culte public est d'abord un culte, si de plus il exige réunion de prières, manifestation commune de sentiments que l'on a dans le cœur, alors au contraire, je l'y trouve très rare et très maigre [...]. Qui peut ignorer en effet que les grandes messes et les vêpres des dimanches ordinaires lesquelles, pour le chant seulement, coûtent aux Fabriques en moyenne 150 francs par dimanche, sont très peu fréquentées et se célèbrent le plus souvent dans le vide [...] malgré le spectacle d'un chœur garni de prêtres ou de gens déguisés en prêtres, malgré la richesse des ornements et la pompe des cérémonies ${ }^{25}$.

En France, un nombre croissant d'architectes provinciaux sont sollicités pour dessiner des vêtements sacrés et surtout certains membres éminents du corps épiscopal soutiennent les artistes et les maisons de fabrication en leur donnant des commandes importantes. La figure dominante est celle de Pierre-Simon de Dreux-Brézé, évêque de Moulins de 1850 à 1893 , troisième fils du marquis de Dreux-Brézé, grand maître des cérémonies à la cour de Louis XVI et d'Adélaïde de Custine. Né le 2 juin 1811, il entre au séminaire

24. Cité par Gérard Cholvy, Histoire religieuse ..., op. cit., p. 131.

25. Anonyme, La Religion est perdue à Paris [...] Lettres d'un vicaire parisien à son évêque en date de 1849 suivies d'un mémoire adressé au même, présentation par Yvan Daniel, Paris, 1991, p. 48-49. 
Saint-Sulpice de Paris et devient prêtre en 1834, après avoir obtenu le doctorat en théologie à Rome. À Paris où il exerce les premières années de son ministère comme grand vicaire de l'archevêque, Mgr de Quélen, il côtoie un grand nombre d'artistes et rencontre en particulier l'orfèvre Poussielgue-Rusand, vers 1840. Il a alors une véritable révélation et comprend que le néogothique sera la meilleure manière de rénover le style de l'Église de France. En 1849, il commande, pour la chapelle du château familial, un ensemble du plus pur style médiéval ${ }^{26}$. Sur la proposition du ministre des cultes, Falloux, il est préconisé évêque de Moulins à la fin de l'année 1849 et sacré à Notre-Dame de Paris, le 7 janvier 1850. Son sacre est l'occasion d'affirmer ses goûts pour le nouveau courant. Dans L'Écho de l'Allier, Anatole d'Auvergne écrit : « Le sacre de Mgr l'évêque de Moulins va devenir le signal d'une réforme que les artistes et les archéologues ont préparé depuis longtemps ${ }^{27}$. 》 Sa chapelle épiscopale est exécutée par Poussielgue-Rusand d'après des dessins de Viollet-le-Duc ${ }^{28}$. Sa mitre est dessinée par Jean-Baptiste Lassus qui s'inspire fortement de celle dite « de saint Thomas Beckett », conservée au Trésor de Sens. Sa chasuble, encore conservée à Moulins serait, d'après les travaux de Jean-Michel Léniaud, elle aussi l'œuvre de Lassus ${ }^{29}$. Pendant son long pontificat, le prélat, resté très « grand seigneur » a enrichi considérablement le vestiaire de sa cathédrale, faisant souvent appel à Hubert Ménage, de Paris. En 1858, il commande un pluvial rouge, tissé par la maison lyonnaise Lemire Père \& Fils (devenue, en 1866, Lamy-Giraud), sur un dessin d'Arthur Martin. En 1879, la même maison lui fournit un « ornement en brocart rouge, brodé d'or », comprenant au moins une chasuble et un pluvial. Il faut noter que les chasubles exécutées pour l'évêque de Moulins, n'ont pas l'ampleur de celles recommandées par Dom Guéranger. Elles couvrent juste les bras, reprenant la forme des chasubles du XVI ${ }^{\mathrm{e}}$ siècle. Ce modèle, qui semble avoir été commercialisé par Lamy \& Giraud, à bon marché ( 85 francs) a reçu l'approbation des liturgistes romains et même du redoutable

26. Guy Massin Le Goff, L'Anjou religieux et les orfèvres du XIXe siècle, Angers, 1983, p. 26.

27. Jean-Michel Léniaud, "Le Trésor néogothique de Moulins», Monuments historiques, Paris, t. III, août 1978, p. 55-58.

28. Idem.

29. Idem. 
Barbier de Montault ${ }^{30}$.

Parmi les autres prélats s'intéressant au néogothique, il faut mentionner l'archevêque de Paris, Marie-Dominique Sibour, peut-être converti par Dreux-Brézé qui lui offre un missel romain dessiné par Lassus. En 1851, pour la fête de Pâques, il arbore une mitre presque semblable à celle de Mgr de Dreux-Brézé, dessinée par Lassus, complétée l'année suivante par un ornement pontifical de forme ample ${ }^{31}$. C'est dans cette tenue que Thomas Couture a peint le prélat sur son lit de mort, en $1857^{32}$. À Lyon, le cardinal de Bonald commande la chasuble « aux anges » dessinée par l'architecte Antoine Desjardins, tissée par Jaillard et brodée chez Bouvard et Lançon. Cette chasuble qui glorifie la sainteté lyonnaise est portée pour la Toussaint 1855. Elle forme un ensemble pontifical avec deux dalmatiques et un pluvial ${ }^{33}$. Dans les décennies qui suivent, à travers les commandes ou les relations de sacre, on constate l'intérêt des évêques pour les formes néogothiques; Mgr Nanquette, du Mans, commande une mitre à Lassus, vers 1860; Mgr Lavigerie, pour son sacre à Rome, une mitre basse qui fait scandale. De même, le nonce d'origine polonaise Wlodojimiez Czarcki, mort en 1888 cardinal prêtre de Sainte-Pudentienne, a été suffisamment marqué par son passage à Paris pour désirer se faire représenter, sur son gisant, vêtu pontificalement à la manière du Xv siècle. À Londres, le cardinal Wiseman, archevêque de Westminster, porte dès 1848 les parements confectionnés par la maison Lonsdale \& Tyler. Sont également très convaincus Antoine de Salinis à Amiens, PierreLouis Parisis à Langres, Casimir Wicard à Fréjus, Thomas Gousset à Reims.

Le mouvement a reçu les encouragements de Lacordaire et de l'ordre dominicain renaissant. Plusieurs chasubles de forme ample ayant appartenu au célèbre dominicain sont conservées ; une, en particulier, offerte par les Dames du Tiers-Ordre de Saint-Dominique, de Toulouse, en 1856, s'inspire directement de la chasuble

30. Xavier Barbier de Montault, «L'Exposition religieuse à Rome », Revue du monde catholique, 1870, vol. 3, p. 589.

31. H. de Riancey, «Du retour aux anciens ornements sacerdotaux, ornements de Mgr l'archevêque de Paris pour la fête de Pâques 1852 », L'Ami de la Religion, t. 156, avril 1852, p. 81-85.

32. Musée Carnavalet, Paris.

33. Bernard Berthod, Élisabeth Hardouin Fugier, Paramentica..., op. cit., p. $174-177$. 
dite de saint Dominique, conservée à Saint-Sernin ${ }^{34}$. Emmanuel d'Alzon, fondateur des augustins de l'Assomption, considère ce mouvement comme indispensable pour cimenter l'unité autour du Saint-Siège. Il fait exécuter tout le vestiaire liturgique du collège de l'Assomption de Nîmes, dans le goût néogothique, d'après des dessins de l'architecte Henry Revoil ${ }^{35}$. À Lyon, les prêtres de Saint-Irénée, responsables de la maison d'éducation dite «des Chartreux », font concevoir le matériel liturgique dans le goût du $\mathrm{XIII}^{\mathrm{e}}$ siècle, pour aller de pair avec la copie de la Sainte-Chapelle que l'architecte Tony Desjardins édifie dans leur propriété de la Croix-Rousse.

Les publications de Didron font très vite des émules parmi les fabricants, surtout les orfèvres. Le premier dessin de Pugin pour le vestiaire liturgique est celui d'une chasuble exécutée en 1846 pour la consécration de l'église de Cheadre ${ }^{36}$. Parmi les vêtements liturgiques dessinés par l'architecte, il faut aussi noter ceux conservés à Saint Chad's Cathedral, taillés dans un riche damas à décor médiéval enrichi de broderies et de plaques d'argent enchâssés ${ }^{37}$. Plusieurs dessins pour des vêtements pontificaux ont été conservés, en particulier celui d'une mitre précieuse pour le cardinal Wiseman, s'inspirant d'une manière manifeste d'une mitre conservée au Trésor royal de Saint-Denis. Les visiteurs de l'exposition de Londres, en 1851, en sentent les prémices. L'exposition de Paris en 1855 permet de constater l'impact du mouvement archéologique sur la création textile et l'orfèvrerie. Didron en témoigne avec fierté, vantant les objets liturgiques réalisés dans le plus pur style néo-médiéval. Il donne également une idée de la chaîne de fabrication : « À Lyon, MM. Jaillard et Le Mire tissent des étoffes que M. Guibout débite à Paris et que MM. Hubert Ménage, Biais, Kreichgauer, Dubus, Limas-Boutron, tous cinq à Paris également, confectionnent en chasubles, chapes, étoles, manipules, mitres et bannières ${ }^{38}$. » Cette pro-

34. "Chasuble du père Lacordaire », Toulouse et l'art médiéval, 1830-1870, Toulouse, $1983, \mathrm{n}^{\circ} 283$.

35. «Didron », Annales archéologiques, Paris, t. xvi, p. 126.

36. Louis de Farcy, La Broderie, op. cit., p. 111.

37. L. Synge, « Ecclesiastical Needlework », The Royal School of Needworks and Embroidery, Londres, 1986, p. 246.

38. Adolphe Napoléon Didron, « Exposition de l'industrie », Annales archéologiques, 1855, t. XV, p. 260. Cité par F. Valentin, L'Art de la soie, Prelle, 17522002, Paris, 2002, p. 134. 
duction est exportée en Afrique du Nord, au Levant, aux États-Unis d'Amérique, en Amérique latine ${ }^{39}$.

\section{LES DIFFICULTÉS DE COMPRÉHENSION AVEC ROME}

On a souvent décrit la position du Saint-Siège comme résolument opposée à l'usage des vêtements de forme ample; plus qu'une opposition, on est en présence d'une léthargie de la Sacrée Congrégation des Rites qui travaille avec une lenteur étonnante. Plusieurs évêques ultramontains en ont fait l'expérience, attendant en vain une approbation de leur missel et ce malgré l'insistance des nonces. La situation est d'ailleurs paradoxale lorsque l'on songe que les précurseurs du mouvement sont des personnalités farouchement ultramontaines. En revanche, aucun texte ne sanctionne la forme et le décor des vases sacrés.

La chasuble de forme ample n'a jamais été totalement abandonnée. La forme ancienne " authentique », est restée en usage dans de nombreux diocèses; les liturgistes l'attestent: Garauti cite le cas de Milan ${ }^{40}$, Farcy cite l'usage du diocèse d'Angers, Migne fait référence à Paris, Narbonne, Toul, Cambrai, $\mathrm{Arras}^{41}$; le chanoine Frézet décrit l'usage de larges chasubles violettes et noires, dans l'église de Reims pendant l'Avent et le Carême ${ }^{42}$. De plus le Cérémonial des évêques, dans sa rubrique sur la vêture, fait explicitement référence au port des chasubles amples : «L'évêque revêt la chasuble que l'on adapte et replie soigneusement de part et d'autre sur les bras ${ }^{43}$. » Des peintures des XVII ${ }^{\mathrm{e}}$ et XVIII ${ }^{\mathrm{e}}$ siècles représentant des événements d'actualité montrent le port contemporain de chasubles amples ${ }^{44}$. Pour étayer la position rigide de Rome, les auteurs citent souvent un texte de la Congrégation des Rites adressé aux

39. Bernard Berthod, «Le renouveau du vêtement liturgique au XIX siècle, l'exemple lyonnais ", Bulletin des Musées et Monuments lyonnais, $\mathrm{n}^{\circ}$ 3, 1999, p. 29.

40. Dictionnaire de droit canonique, t. III, col. 76 .

41. Migne, Dictionnaire de liturgie, Tours, 1849, col. 805.

42. A. Frézet, «La tradition de l'Église de Reims au sujet des ornements de grande forme ", Année Liturgique, t. III, 1916-1917, p. 182-184.

43. Coremoniale Episcoporum, Rome, 1957, livre II, chap. 8, n 19.

44. Par exemple Le Sueur, La Messe de saint Bruno, Paris, musée du Louvre. 
évêques d'Allemagne et de Belgique, dans lequel il est notifié qu'il « n'est permis à personne, sans l'agrément du Saint-Siège, de s'écarter de la forme usuelle reçue dans l'Église, pour en adopter une autre, même ancienne, dite de forme gothique ${ }^{45} »$. En fait, cette position est beaucoup moins stricte que certains ont voulu l'écrire.

Lors de l'apparition des formes médiévales en Europe transalpine, sous le pontificat de Pie IX, de nombreux liturgistes romains se déclarèrent opposés à tout changement et, le centralisme romain aidant, voulurent faire interdire cette nouvelle conception du vêtement liturgique. En 1863, un de ses plus farouches adversaires, Mgr Giovanni Corazza, maître des cérémonies de la Chapelle papale, de retour d'Allemagne où il a constaté ces « abus », écrit un texte très dur que Pie IX ne lui permet pas de publier. En revanche, le pape fait adresser à tous les évêques une circulaire par laquelle il leur demande « de constater l'évolution et de ne pas revenir en arrière ${ }^{46}$ ». En fait, cette circulaire signée par le cardinal Patrizzi, préfet de la Sacrée Congrégation des Rites, ne comporte aucune condamnation des vêtements de forme ample, elle ne donne aucune prescription positive, reconnaissant comme d'obligation le respect du droit coutumier; en revanche, elle condamne l'évêque intervenant de manière autoritaire pour modifier la coutume universelle, troublant ainsi les fidèles. Le même Mgr Corazza, au sacre de Mgr Lavigerie (22 mars 1863), à Rome, exige que ce dernier abandonne sa mitre de style gothique; le nouvel évêque s'en plaint à Pie IX, qui « usant lui-même d'ornements gothiques » blâme le maître des cérémonies ${ }^{47}$. Si la forme a trouvé des adversaires, en revanche, personne ne s'élève contre le retour du tissu à décor symbolique ni contre la reproduction des tissus médiévaux « redécouverts » par le chanoine Bock. Xavier Barbier de Montault est le premier à encourager les fabricants à employer ces tissus plutôt que les « brocarts du XVIII ${ }^{\mathrm{e}}$ siècle $^{48}$ ».

45. Sacrée Congrégation des Rites, circulaire d'août 1863, in Acta juris pontificae, 1864, col. 627-628. La circulaire est reprise à l'identique le 9 décembre 1925.

46. Xavier Barbier de Montault, Traité de la construction, de l'ameublement et de la décoration des églises selon les règles canoniques et les traditions romaines, Paris, 1877 , t. II, p. 330-334.

47. Jean Deshusses, "Mitre», in Dictionnaire de droit canonique, Paris, 1957, t. VI, col. 917.

48. Xavier Barbier de Montault, «L'Exposition religieuse à Rome... », art. cit., p. 738. 
La position de Rome, sans être très claire, n'est donc pas défavorable au retour des formes médiévales, même si, dans une grande partie du monde catholique, en Amérique latine, en Espagne, et en Italie surtout, la forme héritée du concile de Trente reste en usage. À l'exception de la circulaire du cardinal Patrizzi, aucun texte pendant soixante ans ne fait allusion à ce problème, ce qui peut être interprété comme un "édit de tolérance », selon le mot de Deshusses. En 1925, une importante exposition d'art sacré est organisée à Rome, sous l'autorité du Saint-Siège; la plupart des chasubles exposées sont de forme ample, aussi dans ce contexte, la circulaire de la sacrée Congrégation des Rites déjà citée et reprise le 9 décembre de la même année apparaît comme un ultime combat d'arrière-garde ${ }^{49}$.

\section{LE CAS ANGLICAN}

Le mouvement néogothique n'a pas concerné que le catholicisme : l'Église d'Angleterre notamment n'a jamais abandonné les vêtements de forme médiévale. Aux XvII ${ }^{\mathrm{e}}$ et XvIII ${ }^{\mathrm{e}}$ siècles, l'influence des milieux puritains a cependant entraîné un net recul de l'utilisation du matériel liturgique. Le Gothic Revival anglais coïncide avec un retour aux pratiques rituelles dans la Haute Église (Hight Church). August Pugin a été suivi par d'autres architectes, par exemple Sir Arthur Blomfield, auteur de la Radecliffe Infirmary Chapel à Oxford, qui a dessiné pour le cardinal Newman. La firme de Birmingham Jones \& Willis a popularisé le vêtement néogothique par la confection à bon marché, soutenue par l'édition de catalogues commerciaux. La Haute Église, en retrouvant les costumes et le décor antérieurs à la Réforme, tente, peut-être inconsciemment, d'effacer les traits les plus violents de cette dernière. Au Xx ${ }^{e}$ siècle, elle conserve les formes médiévales, axant sa recherche sur le décor sans hésiter à faire des choix très contemporains - l'illustration la plus spectaculaire demeurant le célèbre pluvial pour l'archevêque de Canterbury, lors du Jubilé de la reine Elisabeth II, en

49. Ce qui n'empêche pas le très conservateur cardinal Maurin, archevêque de Lyon, de demander en 1927, la «permission de se servir des ornements gothiques dans tout le diocèse ", permission accordée par le cardinal Vico, évêque de Porto et préfet de la Congrégation des Rites, le 3 novembre 1927 (archives de l'Archevêché de Lyon, 10.II.7 (4)). 
$1977^{50}$. En revanche, dès 1931, Louisa Pesel rassemble une centaine de brodeuses amateurs autour de la cathédrale de Winchester et travaille sur des dessins des XVI ${ }^{\mathrm{e}}$ et $\mathrm{XVII}{ }^{\mathrm{e}}$ siècles.

\section{DéClin OU Renouveau?}

Le néogothique liturgique est cependant un échec au point de vue apostolique car le mouvement ne touche que les intellectuels. Les fidèles apprécient le style de piété ultramontaine, le culte de Marie, du Sacré-Cœur, les neuvaines, les indulgences mais ils regrettent l'abandon du culte des saints locaux. Ce sont davantage les grandioses cérémonies déployant un grand nombre d'officiants que la beauté de la liturgie elle-même qui touchent les foules. D'autant que les grands rassemblements après 1880 dépassent la sphère du religieux et deviennent de véritables manifestes politiques. À la mort des grands évêques « néogothiques », on ne constate pas de filiation. Leurs successeurs polarisent leur énergie dans le conflit avec l'État qui s'établit dans de nombreux pays d'Europe. Ainsi, en France, l'expérience de Solesmes et des autres monastères bénédictins est interrompue par l'exil des moines en 1901. La dimension politique de la lutte prime sur le développement d'une réelle réflexion sur la liturgie. Il faut attendre le règne de Pie $\mathrm{X}$ pour recevoir des textes romains concernant la pratique dominicale, la célébration eucharistique, avec l'encouragement du chant grégorien.

Les témoins iconographiques de l'époque, peintures et surtout photographies, attestent du manque de grandeur des cérémonies religieuses. On constate la présence pléthorique du clergé vêtu de manière hétéroclite en l'absence de grands ensembles, avec des vêtements liturgiques disparates, les évêques en mozette arborant la mitre à la place de la modeste barrette ${ }^{51}$, créant ainsi un costume hybride, ni habit de chœur ni vêtement liturgique, qui leur paraissait plus fastueux! Les photographies prises à Paray-le-Monial, à Lourdes pour la consécration de la basilique, à Fourvière pour celle de l'église votive (1896) témoignent de cet hétéroclisme où l'esthétique liturgique est niée au profit du grandiloquent.

50. Beryl Dean, Embroidery in Religion and Ceremonial, Londres, 1985, p. $9-88$

51. D'autant que la barrette violette n'est autorisée que depuis 1888. 
Après 1890 , le néogothique commence à lasser, contrairement à ce qu'avaient rêvé les pionniers du mouvement, lorsqu'Arthur Martin écrivait avec enthousiasme : «On abandonne presque partout, aujourd'hui, cette forme étriquée du XVIII ${ }^{\mathrm{e}}$ siècle pour se rapprocher de l'usage des anciens fidèles ${ }^{52}$. » Les grandes maisons de soierie lyonnaises se retrouvent, à la fin du siècle, avec d'importants stocks d'étoffes qu'elles n'ont pas vendues ${ }^{53}$. La forme étriquée et souvent mal taillée, tant critiquée par Didron, n'a jamais cessé d'être produite ni de plaire. Les catalogues de fournisseurs et la grande quantité de chasubles de forme tridentine conservée dans les sacristies, attestent cet échec. À la fin du pontificat de Léon XIII (1903), les principales maisons de paramentique proposent à leur clientèle quelques modèles amples à côté de modèles " romains " qui sont souvent loin de l'usage romain, comme le note Xavier Barbier de Montault. L'Église d'Angleterre a été plus fidèle à Pugin.

$\mathrm{Au}$ début $\mathrm{du} \mathrm{xx}^{\mathrm{e}}$ siècle, une réflexion s'élabore autour de quelques artistes et religieux. En France, Maurice Denis et Georges Desvallières fondent les Ateliers d'art sacré (1919) et Paul CroixMarie les Artisans de l'autel (1918). La réflexion sur l'art liturgique conduit les théoriciens à rechercher des formules plus « authentiques » car la multiplication industrielle gène la visibilité du travail de l'artiste. Le vêtement ou le vase sacré sont d'abord conçus comme une œuvre d'art et une prière, non comme un objet confectionné en série et vendu par correspondance. La broderie cède devant l'application d'étoffe déjà conseillée par Arthur Martin. Mais les maisons de confection ne suivent pas cette nouvelle perception du vêtement, qui reste limitée à de petits cercles, et continuent à produire des chasubles de toutes formes tout en privilégiant de plus en plus la forme ample. On note ainsi un recul de la paramentique de qualité dans la production industrielle au profit de petits ateliers artisanaux. Le métal est travaillé au marteau et au ciseau, les émaux disparaissent au profit de l'ivoire et de l'incrustation de pierres.

En France et dans toute l'Europe, des groupes composés d'artistes et de clercs créent des ateliers avec le désir de redonner au vêtement liturgique sa dimension sacrée. La famille bénédictine, fidèle à la

52. Christine Aribaud, «L'étoffe des solennités : la soie en service dans les églises du Tarn ( $\mathrm{xv}^{\mathrm{e}}-\mathrm{xx} \mathrm{x}^{\mathrm{e}}$ siècle) », Textiles sacrés du Tarn, Paris, 2003, p. 35.

53. Florence Valentin, L'Art de la soie..., op. cit., p. 135. 
mémoire de Dom Guéranger, tient une large place dans ce renouveau. En Allemagne, l'abbaye de Maria-Laach, fondée en 1892, devient un grand centre d'étude, de conception et de fabrication. Son premier abbé Dom Maur Wolter, émule de Dom Didier Lenz, le grand maître de l'École de Beuron, groupe autour de lui quelques moines-artistes, tel frère Nicolas Becker, peintre et dessinateur de vêtements et frère Radbod Commandeur qui créera l'atelier d'orfèvrerie de l'abbaye en 191154. À Solesmes, Dom de La Borde et Dom Lecore créent des ensembles en utilisant des soieries lyonnaises qui sont assemblées sur place. Entre les deux guerres, un groupe de moniales crée l'Atelier des Religieuses missionnaires de Saint-Benoît dont la mission est précisément l'artisanat liturgique.

Dans cet esprit, le styliste liturgique Stefano Zanella (Trévise) travaille, à partir de 1990, pour introduire la tradition médiévale à Rome $^{55}$. La forme de la chasuble qu'il propose couvre tout le corps comme celle en usage à la fin du premier millénaire. Il agit de même pour les mitres. Jusqu'au début du pontificat de Jean-Paul II, la mitre prélatice était particulièrement haute, pouvant atteindre 60 centimètres. Le tailleur trévisan propose une forme basse qui a été adoptée pour les mitres papales et cardinalices portées aux chapelles papales.

Si l'idéal de beauté, copié du XIII ${ }^{\mathrm{e}}$ siècle, n'a pas converti les foules, il a permis un renouveau de l'objet sacré et une reprise en compte du rôle de l'artiste dans sa conception. Le vêtement de forme ample s'est installé de façon durable et a conquis la cour romaine à la fin $\mathrm{du} \mathrm{xx}^{\mathrm{e}}$ siècle. Piero Marini, le maître des célébrations liturgiques de Jean-Paul II, en était bien conscient lorsqu'il demanda à Stefano Zanella de créer pour le pape des vêtements «qui doivent manifester l'harmonie et la beauté [...] et retrouver la noble simplicité des temps anciens ${ }^{56} \gg$.

17, rue de la Charité

69002 Lyon

bernardberthod@yahoo.fr

54. L'Artisan liturgique, abbaye de Saint-André, 1931, $\mathrm{n}^{\circ}$ 20, p. 418-423 et $\mathrm{n}^{\circ} 21$, p. 415.

55. Bernard Berthod, Ils habillent le pape, Lyon, Fourvière, 2005.

56. Piero Marini, Liturgia e bellezza, esperienze di rimovamento in alcune celebrazioni pontificie, Rome, Cité du Vatican, 2004 et communication à l'auteur. 